\title{
Relativistic spectral function of nucleon in hot nuclear matter
}

\author{
Sabyasachi Ghosh ${ }^{*}$ and Sourav Sarka1 ${ }^{\dagger}$ \\ Variable Energy Cyclotron Centre, 1/AF, Bidhannagar, Kolkata, 700064, India \\ S. Mallil㞅 \\ Theory Division, Saha Institute of Nuclear Physics, 1/AF Bidhannagar, Kolkata 700064, India
}

(Dated: September 24, 2021)

\begin{abstract}
We present a simple calculation of the nucleon self-energy in nuclear matter at finite temperature in a relativistic framework, using the real time thermal field theory. The imaginary parts of oneloop graphs are identified with discontinuities across the unitary and the Landau cuts. We find that in general both the cuts contribute significantly to the spectral function in the region of (virtual) nucleon mass usually considered, even though the unitary cut is ignored in the literature. Also our relativistic spectral function differs from the one in non-relativistic approximation, used in some earlier calculations.
\end{abstract}

Heavy ion collisions provide an opportunity to investigate particle propagation through strongly interacting media. However, only the vector mesons, particularly the $\rho$, can at present be studied directly by detecting dileptons, into which they decay in the hot, dense media. The media created by these collisions consist, in general, not only of mesons, but also of nucleons. Thus the effects of both mesons and nucleons on the vector meson spectral functions have been extensively studied in the literature [1]. For a more complete picture, the self-energy of nucleon itself need be investigated [2, 3]. The nucleon self-energy function also determines the equation of state of nuclear matter [4].

In this work, we find the one loop corrections to the nucleon propagator at finite temperature and nucleon chemical potential in the real time formulation of the thermal field theory [5]. However, to keep an eventual contact with the fundamental $Q C D$ theory, we do not start directly with the propagator, namely the two point function of the nucleon field. Instead, we consider the same of the nucleon current $\eta(x)$ [6, 7], which in vacuum is

$$
i \int d^{4} x e^{i p \cdot x}\langle 0|T \eta(x) \bar{\eta}(0)| 0\rangle \text {. }
$$

Here $\eta(x)$ is built out of three quark fields, so as to have the quantum numbers of the nucleon. We denote its matrix element between vacuum and nucleon state as

$$
\langle 0|\eta(x)| N(p)\rangle=\lambda u(p) e^{i p \cdot x},
$$

where $u(p)$ is the Dirac spinor of the nucleon and the parameter $\lambda$ denotes the coupling of $\eta(x)$ with the nucleon [8].

In nuclear matter, the vacuum expectation value in Eq. (1) must be replaced by the ensemble average. Denoting the time-ordered product of the operators by $O(x)$, it means

$$
\langle 0|O(x)| 0\rangle \rightarrow \operatorname{Tr}\left[e^{-\beta(H-\mu N)} O(x)\right] / Z, \quad Z=\operatorname{Tr}\left[e^{-\beta(H-\mu N)}\right],
$$

where $H$ and $N$ are the Hamiltonian and the nucleon number operator of the system , $\beta$ is inverse temperature and $\mu$ is the chemical potential corresponding to $N$. In the real time version, every two point function, including the self-energy we calculate below, assumes the form of a $2 \times 2$ matrix. But each of these matrices may be diagonalised, when it is given essentially by single analytic function, that determines completely the dynamics of the corresponding two-point function [9]. As this function is simply related to any one, say the 11-component of the matrix, we need calculate only this component of the self-energy matrix.

The 11-component of a free, thermal matrix propagator for a particle is a sum of its vacuum propagator and a term depending on the (on-shell) distribution functions of like-particles in the medium through which it propagates. The latter term has a universal form, depending only on the bosonic or the fermionic character of the particle [10]. Anticipating the propagators for pion, nucleon and $\Delta(1237)$ to appear in our calculation, we begin by writing their 11-components. These particles are represented respectively by scalar $(\phi(x))$, Dirac $(\psi(x))$ and Rarita-Schwinger $\left(\Delta_{\mu}(x)\right)$ fields [1]. For the bosonic propagator, it is

$$
D^{11}\left(k_{0}, \vec{k}, m_{\pi}\right)=\Delta\left(k, m_{\pi}\right)+2 \pi i n(\omega) \delta\left(k^{2}-m_{\pi}^{2}\right),
$$

\footnotetext{
*Electronic address: sabyasachighosh@veccal.ernet.in

${ }^{\dagger}$ Electronic address: sourav@veccal.ernet.in

${ }^{\ddagger}$ Electronic address: mallik@theory.saha.ernet.in
} 


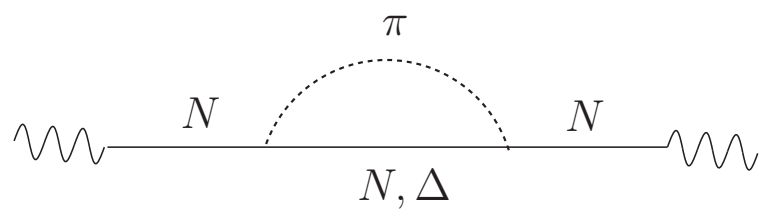

FIG. 1: One-loop graphs for the two-point function contributing to self-energy of the nucleon

where $\Delta$ and $n$ are the vacuum propagator of the scalar field and its equilibrium particle distribution function,

$$
\Delta\left(k, m_{\pi}\right)=\frac{-1}{k^{2}-m_{\pi}^{2}+i \eta}, \quad n(\omega)=\frac{1}{e^{\beta \omega}-1}, \quad \omega=\sqrt{\vec{k}^{2}+m_{\pi}^{2}} .
$$

For the fermionic propagators, we introduce

$$
E^{11}\left(p_{0}, \vec{p}, m\right)=\Delta(p, m)-2 \pi i N\left(p_{0}\right) \delta\left(p^{2}-m^{2}\right)
$$

where $N\left(p_{0}\right)$ consists of distribution functions for the particle and anti-particle,

$$
N\left(p_{0}\right)=n_{+}\left(\omega^{\prime}\right) \theta\left(p_{0}\right)+n_{-}\left(\omega^{\prime}\right) \theta\left(-p_{0}\right), \quad n_{ \pm}\left(\omega^{\prime}\right)=\frac{1}{e^{\beta\left(\omega^{\prime} \mp \mu\right)}+1}, \quad \omega^{\prime}=\sqrt{\boldsymbol{p}^{2}+m^{2}}
$$

in terms of which the 11-components of spin $\frac{1}{2}$ and $\frac{3}{2}$ propagators may be written respectively as

$$
\begin{aligned}
& S^{11}\left(p_{0}, \vec{p}\right)=\left(\not p+m_{N}\right) E^{11}\left(p_{0}, \vec{p}, m_{N}\right) \\
& S_{\mu \nu}^{11}\left(p_{0}, \vec{p}\right)=\left(\not p+m_{\Delta}\right)\left\{-g_{\mu \nu}+\frac{2}{3 m_{\Delta}^{2}} p_{\mu} p_{\nu}+\frac{1}{3} \gamma_{\mu} \gamma_{\nu}+\frac{1}{3 m_{\Delta}}\left(\gamma_{\mu} p_{\nu}-\gamma_{\nu} p_{\mu}\right)\right\} E^{11}\left(p_{0}, \vec{p}, m_{\Delta}\right)
\end{aligned}
$$

The spectral function of nucleon is obtained from the Dyson equation, giving the complete propagator $\boldsymbol{S}^{\prime}$ in terms of the free propagator $\boldsymbol{S}$ and self-energy $\boldsymbol{\Sigma}$,

$$
S^{\prime}=S-S \Sigma S^{\prime}
$$

where each element is a $2 \times 2$ matrix in the thermal indices (besides being $4 \times 4$ matrices in Dirac space). As already stated, they can all be diagonalised to get the respective analytic functions, denoted by a bar, satisfying

$$
\overline{S^{\prime}}=\bar{S}-\bar{S} \bar{\Sigma} \overline{S^{\prime}}
$$

which may be readily solved as usual. The self-energy function is related to the 11-component of the corresponding matrix by [9],

$$
\begin{aligned}
& \operatorname{Re} \bar{\Sigma}(p)=\operatorname{Re} \Sigma^{11}(p) \\
& \operatorname{Im} \bar{\Sigma}(p)=\operatorname{coth}\left[\beta\left(p_{0}-\mu\right) / 2\right] \operatorname{Im} \Sigma^{11}(p)
\end{aligned}
$$

The free propagator $\bar{S}$ turns out to be the same as in vacuum,

$$
\bar{S}(p)=\frac{-\left(\not p+m_{N}\right)}{p^{2}-m_{N}^{2}+i \eta}
$$

The calculation simplifies if we take $\boldsymbol{p}=0$. Also restricting to the anti-nucleon pole in Eq.(13), it becomes

$$
\bar{S}\left(p_{0}\right)=\frac{\left(1+\gamma_{0}\right)}{2} \frac{-1}{p_{0}-m_{N}+i \eta}
$$

Decomposing $\bar{\Sigma}$ and $\bar{S}^{\prime}$ in Dirac space,

$$
\bar{\Sigma}=\Sigma_{s}+\gamma_{0} \Sigma_{v}, \quad \bar{S}^{\prime}=S_{s}^{\prime}+\gamma_{0} S_{v}^{\prime},
$$




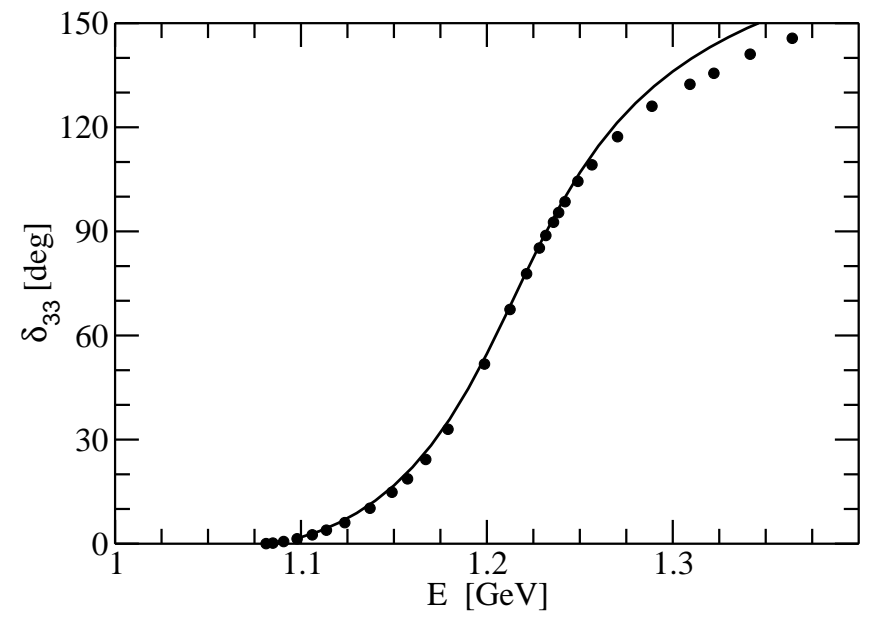

FIG. 2: Graph of phase shift for $P_{33}$ partial wave in $\pi N$ scattering from our model compared with experiment [20] (solid circles)

it follows from Dyson equation that $S_{s}^{\prime}=S_{v}^{\prime}$. Then letting $\Sigma=\Sigma_{s}+\Sigma_{v}$, we get the complete propagator as

$$
\bar{S}^{\prime}\left(p_{0}\right)=\frac{\left(1+\gamma_{0}\right)}{2} \frac{-1}{p_{0}-m_{N}-\Sigma}
$$

giving the spectral function

$$
A_{N}\left(p_{0}\right)=\frac{-\operatorname{Im} \Sigma}{\left(p_{0}-m_{N}-\operatorname{Re} \Sigma\right)^{2}+(\operatorname{Im} \Sigma)^{2}}
$$

The graphs which we wish to evaluate are shown in Fig. 1. Because we are interested in finding the nucleon selfenergy, we retain only the graphs which couple the nucleon current to the nucleon. Also we include $\Delta(1237)$ resonance besides the nucleon in the intermediate state. We shall comment later on the contribution of higher resonances.

The vertices appearing in Fig.1 may be obtained from chiral perturbation theory [12 14]. The appropriate field variable for pion in the effective theory is not $\phi(x)$ introduced earlier, but the $S U(2)$ valued matrix field $u(x)$ related to $\phi(x)$ by $u(x)=\exp \left(i \vec{\tau} \cdot \vec{\phi} / 2 F_{\pi}\right)$, where $F_{\pi}=93 \mathrm{MeV}$, the so-called pion decay constant. The effective Lagrangians are [15],

$$
\begin{aligned}
\mathcal{L}_{\pi N N} & =\frac{1}{2} g_{A} \bar{\psi} \psi \gamma_{5} \psi=-\frac{g_{A}}{F_{\pi}} \bar{\psi} \gamma_{\mu} \gamma_{5} \tau^{a} \psi \partial^{\mu} \phi_{a}+\cdots \\
\mathcal{L}_{\pi N \Delta} & =\frac{g_{\Delta}}{\sqrt{2}} \bar{\psi}_{a}\left(u^{\mu}\right)_{b}^{c} \Delta_{\mu}^{a b d} \epsilon_{c d}+\text { h.c. }=\frac{g_{\Delta}}{F_{\pi}} \bar{p} \partial^{\mu} \pi^{-} \Delta_{\mu}^{++}+\cdots
\end{aligned}
$$

where $u_{\mu}=i\left(u^{\dagger} \partial_{\mu} u-u \partial_{\mu} u^{\dagger}\right), a, b \cdots=1,2$ and $\epsilon_{12}=-\epsilon_{21}=1$, etc. The coupling constants $g_{A}$ and $g_{\Delta}$ are to be determined phenomenologically. As is well-known [17, 18], such a model requires form factors at the vertices, which we take in the Lorentz invariant form as

$$
F(p, k)=\frac{\Lambda^{2}}{\Lambda^{2}+\left(p \cdot k / m_{N}\right)^{2}-k^{2}}
$$

where $p$ and $k$ are the four-momenta of nucleon and pion at the vertices and $\Lambda$ is essentially a cut-off on these momenta.

We first check this model with experimental data on $\pi N$ scattering. The interaction (19) allows us to calculate the decay width of $\Delta \rightarrow N+\pi$ as a function of its energy as

$$
\Gamma(E)=\frac{1}{24 \pi}\left(\frac{g_{\Delta}}{F_{\pi}}\right)^{2} F^{2}(E)|\boldsymbol{p}|^{3} \frac{\left(E+m_{N}\right)^{2}-m_{\pi}^{2}}{E^{2}}
$$

Here $\boldsymbol{p}$ is the three-momentum in the centre-of-mass of $\pi N$ system,

$$
\boldsymbol{p}^{2}=\frac{\left\{E^{2}-\left(m_{N}+m_{\pi}\right)^{2}\right\}\left\{E^{2}-\left(m_{N}-m_{\pi}\right)^{2}\right\}}{4 E^{2}} .
$$




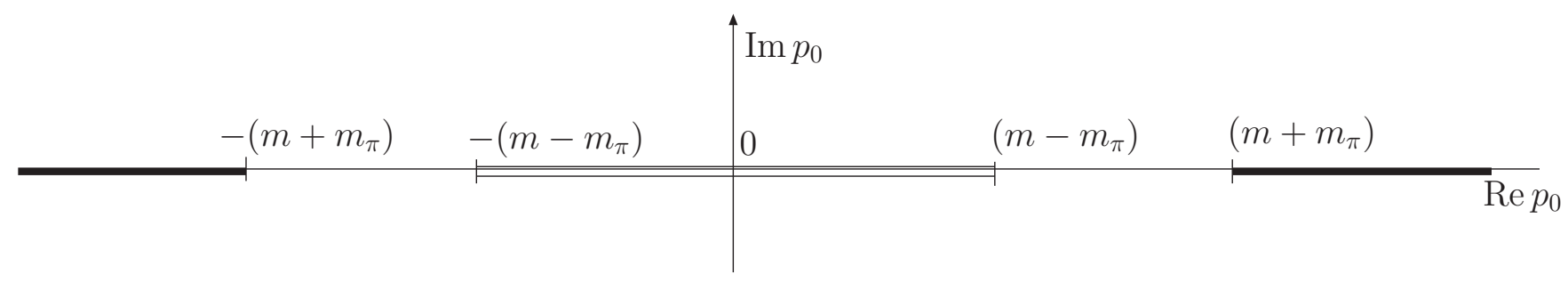

FIG. 3: Branch cuts of self-energy function in $p_{0}$ plane for $\boldsymbol{p}=0$.

In this kinematic configuration, the form factor becomes

$$
F(|\boldsymbol{p}|)=\frac{\Lambda^{2}}{\Lambda^{2}+\left(|\boldsymbol{p}| E / m_{N}\right)^{2}}
$$

The pion-nucleon partial wave $f$ in the $P_{33}$ channel may now be written in the form

$$
f(E) \sim \frac{1}{E^{2}-m_{\Delta}^{2}+i m_{\Delta} \Gamma(E)}
$$

We take the resonance parameters at the pole position, $m_{\Delta}=1210 \mathrm{MeV}$ and $\Gamma\left(m_{\Delta}\right)=100 \mathrm{MeV}$ [19]. Taking $g_{\Delta}=2.2$ and $\Lambda=400 \mathrm{MeV}$ [18], we can satisfy Eq.(21) and also achieve reasonable agreement of the phase shift $\delta_{33}$ computed from Eq.(24) with experiment [20] (Fig. 2). Also we take $g_{A}=1.26$ [21] and the same form factor at the $\pi N N$ vertex. form

We now evaluate the self-energies from graphs of Fig. 1. The 11-component of each of the loops has the general

$$
\Sigma^{11}\left(p_{0}, \boldsymbol{p}\right)=i \int \frac{d^{4} k}{(2 \pi)^{4}} R(p, k) D^{11}(k) E^{11}(p-k)
$$

where $R(p, k)$ includes the form factors and the factor from interaction Lagrangian at the vertices, as well as the spin-dependent factor in the propagator, all of which we shall write below explicitly. Inserting propagators from Eqs.(4) and (6) in Eq.(25), we get

$$
\begin{aligned}
\Sigma^{11}\left(p_{0}, \boldsymbol{p}\right)= & i \int \frac{d^{4} k}{(2 \pi)^{4}} \frac{R\left(k_{0}\right)}{\left(k^{2}-m_{\pi}^{2}+i \epsilon\right)\left\{(p-k)^{2}-m^{2}+i \epsilon\right\}} \\
& -\int \frac{d^{4} k}{(2 \pi)^{3}} R\left(k_{0}\right)\left\{\frac{N\left(p_{0}-k_{0}\right) \delta\left((p-k)^{2}-m^{2}\right)}{k^{2}-m_{\pi}^{2}+i \epsilon}-\frac{n\left(k_{0}\right) \delta\left(\left(k^{2}-m_{\pi}^{2}\right)\right.}{(p-k)^{2}-m^{2}+i \epsilon}\right\} \\
& +i \int \frac{d^{4} k}{(2 \pi)^{2}} R\left(k_{0}\right) n\left(k_{0}\right) N\left(p_{0}-k_{0}\right) \delta\left(k^{2}-m_{\pi}^{2}\right) \delta\left((p-k)^{2}-m^{2}\right)
\end{aligned}
$$

where $m$ denotes mass of baryon $\left(m_{N}\right.$ or $\left.m_{\Delta}\right)$ in the loop and we show the dependence of $R$ on $k_{0}$ only, suppressing other variables for brevity. Here the first term refers to vacuum. The second and the third terms are medium dependent, with distribution functions appearing respectively linearly and quadratically. Observe that the third term is purely imaginary.

Carrying out the $k_{0}$ integration in all the three terms of Eq. (26), we can easily find the real and the imaginary parts of $\Sigma^{11}$. Though $\operatorname{Im} \Sigma^{11}$ contains quadratic terms in distribution functions, $\operatorname{Im} \bar{\Sigma}$, as defined by Eq. (12), turns out to be linear in them [9, 22],

$$
\begin{aligned}
& \operatorname{Im} \bar{\Sigma}\left(p_{0}, \boldsymbol{p}\right)=-\pi \int \frac{d^{3} \boldsymbol{k}}{(2 \pi)^{3} 4 \omega \omega^{\prime}} \times \\
& {\left[R\left(k_{0}=\omega\right)\left\{\left(1+n(\omega)-n_{+}\left(\omega^{\prime}\right)\right) \delta\left(p_{0}-\omega-\omega^{\prime}\right)-\left(n(\omega)+n_{-}\left(\omega^{\prime}\right)\right) \delta\left(p_{0}-\omega+\omega^{\prime}\right)\right\}\right.} \\
& +R\left(k_{0}=-\omega\right)\left\{\left(n(\omega)+n_{+}\left(\omega^{\prime}\right)\right) \delta\left(p_{0}+\omega-\omega^{\prime}\right)-\left(1+n(\omega)-n_{-}\left(\omega^{\prime}\right) \delta\left(p_{0}+\omega+\omega^{\prime}\right)\right\}\right]
\end{aligned}
$$

where $\omega$ and $\omega^{\prime}$ are pion and baryon energies,

$$
\omega=\sqrt{m_{\pi}^{2}+\boldsymbol{k}^{2}}, \quad \omega^{\prime}=\sqrt{m^{2}+(\boldsymbol{p}-\boldsymbol{k})^{2}}
$$



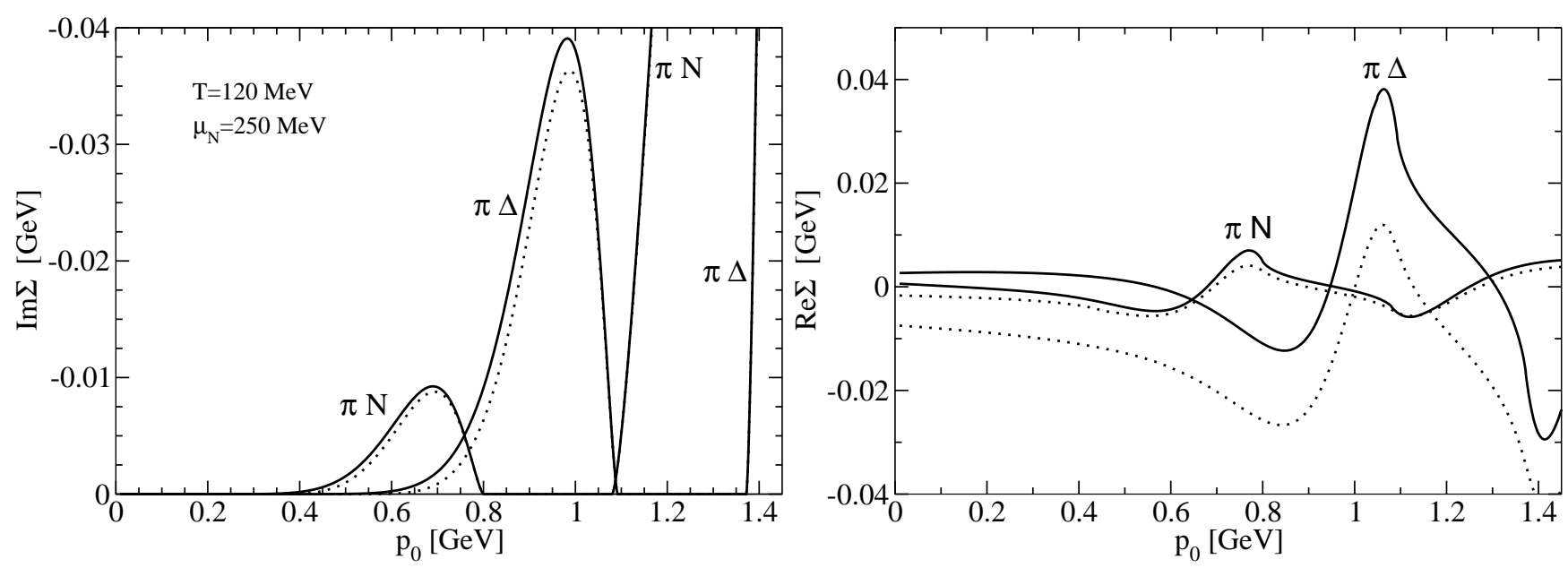

FIG. 4: Imaginary (left panel) and real (right panel) parts of self-energy from $\pi N$ and $\pi \Delta$ loops. Solid curves represent the results of our calculation (relativistic, including unitary cuts). Dotted curves result from non-relativistic approximation.

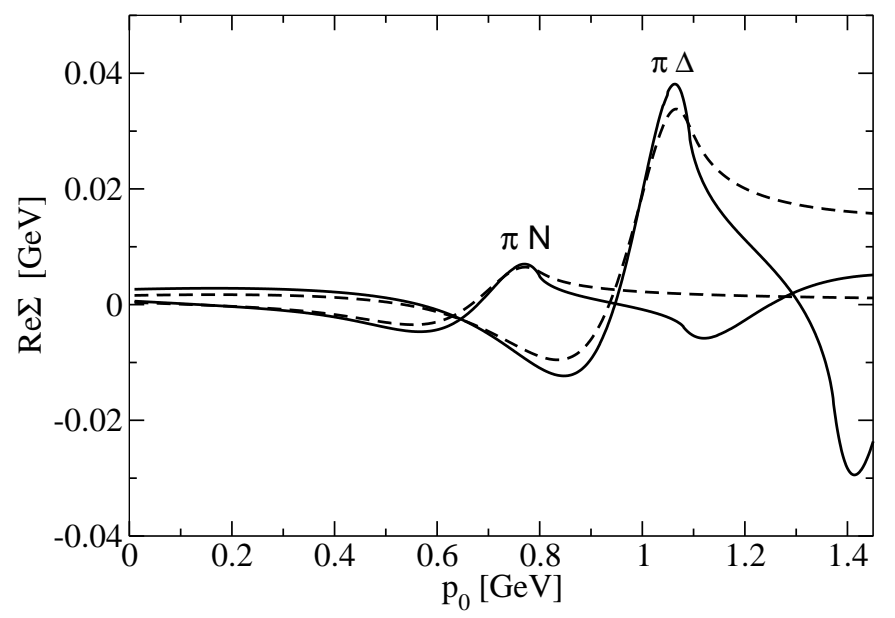

FIG. 5: Real part of nucleon self-energy with (solid) and without (dashed) contribution from the unitary cut.

For the real part of $\bar{\Sigma}$, we include only the medium dependent pieces, which are given by the second term in Eq. (26) alone,

$$
\begin{aligned}
\operatorname{Re} \bar{\Sigma}\left(p_{0}, \boldsymbol{p}\right)=\int \frac{d^{3} \boldsymbol{k}}{(2 \pi)^{3} 4 \omega \omega^{\prime}} & \left\{\frac{R_{2} n+R_{3} n_{+}}{p_{0}+\omega-\omega^{\prime}}+\frac{R_{1} n-R_{3} n_{+}}{p_{0}-\omega-\omega^{\prime}}\right. \\
& \left.-\frac{R_{1} n+R_{4} n_{-}}{p_{0}-\omega+\omega^{\prime}}-\frac{R_{2} n-R_{4} n_{-}}{p_{0}+\omega+\omega^{\prime}}\right\}
\end{aligned}
$$

where $R_{i},(i=1, \cdots, 4)$ denote the function $R\left(k_{0}\right)$ evaluated at $k_{0}=\omega,-\omega, p_{0}-\omega^{\prime}$ and $p_{0}+\omega^{\prime}$ respectively.

In the following we restrict our evaluation to $\boldsymbol{p}=0$, when there will be no angular dependence. First consider the imaginary part, giving the cut structure [23, 24]. The delta-functions in the different terms of Eq. (27) control the regions of non-vanishing imaginary parts of $\bar{\Sigma}$, which define the position of the branch cuts. As shown in Fig. 3, the first and the fourth terms give rise to the regions, $p_{0} \geq\left(m+m_{\pi}\right)$ and $p_{0} \leq-\left(m+m_{\pi}\right)$ respectively, giving the unitary cuts. Similarly the second and the third terms lead to the regions, $0 \leq p_{0} \leq\left(m-m_{\pi}\right)$ and $-\left(m-m_{\pi}\right) \leq p_{0} \leq 0$, giving the Landau cuts.

With $\boldsymbol{p}=0$, the value of $|\boldsymbol{k}|$, fixed by the $\delta$-functions in Eq. (27), is the magnitude of three-momentum in the center-of-mass of the pion-baryon system,

$$
|\boldsymbol{k}|^{2}=\frac{\left\{p_{0}^{2}-\left(m+m_{\pi}\right)^{2}\right\}\left\{p_{0}^{2}-\left(m-m_{\pi}\right)^{2}\right\}}{4 p_{0}^{2}}
$$



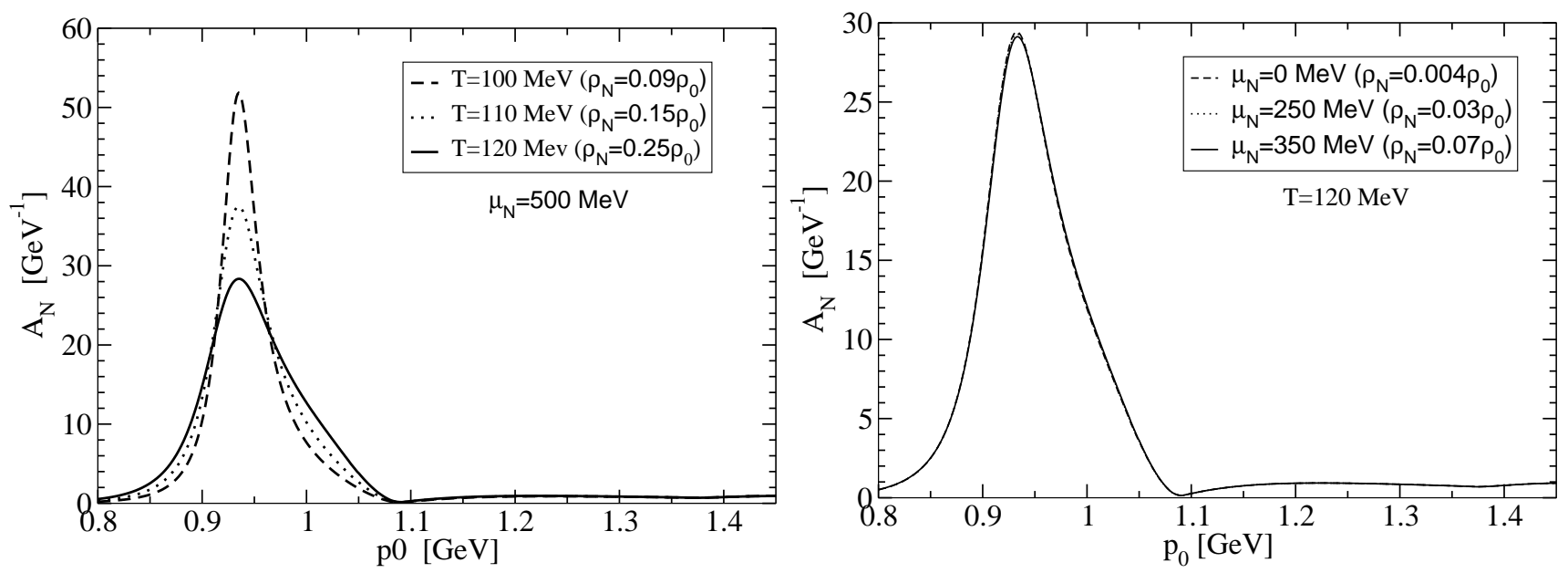

FIG. 6: Nucleon spectral function at different temperatures for a fixed chemical potential (left panel) and at different chemical potentials for a fixed temperature (right panel)

In this frame, the form factor (23) simplifies to

$$
F(|\boldsymbol{k}|)=\frac{\Lambda^{2}}{\Lambda^{2}+\boldsymbol{k}^{2}}
$$

Let us define the variables,

$$
\bar{\omega}=\frac{p_{0}^{2}+m_{\pi}^{2}-m^{2}}{2 p_{0}}, \quad \bar{\omega}^{\prime}=\frac{p_{0}^{2}-m_{\pi}^{2}+m^{2}}{2 p_{0}}
$$

which actually coincide respectively with $\omega$ and $\omega^{\prime}$ defined by Eq.(28) on all the cuts, except for $\omega$ on the Landau cut, where $\omega=|\bar{\omega}|$. Then the imaginary parts of $\bar{\Sigma}$ for $p_{0} \geq 0$ are given by the first and the third terms of Eq.(27) as

$$
\operatorname{Im} \bar{\Sigma}\left(p_{0}\right)=-\frac{|\boldsymbol{k}|}{8 \pi p_{0}} \begin{cases}R\left(k_{0}=\bar{\omega}\right)\left\{1+n(\bar{\omega})-n_{+}\left(\bar{\omega}^{\prime}\right)\right\}, & \text { on unitary cut } \\ R\left(k_{0}=\bar{\omega}\right)\left\{n(|\bar{\omega}|)+n_{+}\left(\bar{\omega}^{\prime}\right)\right\}, & \text { on Landau cut }\end{cases}
$$

The expression (29) for the real part also simplifies for $\boldsymbol{p}=0$ to

$$
\operatorname{Re} \bar{\Sigma}\left(p_{0}\right)=-\frac{1}{16 \pi^{2} p_{0}} P \int_{m_{\pi}^{2}}^{\infty} \frac{d \omega^{2} \sqrt{\omega^{2}-m_{\pi}^{2}} h(\omega)}{\omega \omega^{\prime}\left(\omega^{2}-\bar{\omega}^{2}\right)}
$$

where

$$
h(\omega)=\omega^{\prime}\left\{(\omega+\bar{\omega}) R_{1}-(\omega-\bar{\omega}) R_{2}\right\} n-\omega\left\{\left(\omega^{\prime}+\bar{\omega}^{\prime}\right) R_{3} n_{+}-\left(\omega^{\prime}-\bar{\omega}^{\prime}\right) R_{4} n_{-}\right\}
$$

Having carried out the evaluation in terms of the (Dirac) matrix-function $R(p, k)$, it remains to write its explicit expressions for the two loops,

$$
\begin{aligned}
& R(p, k)=\frac{3}{4}\left(\frac{g_{A}}{F_{\pi}}\right)^{2} F^{2}(p, k)\left\{2 k \cdot p \not k-k^{2}\left(\not p+\not k+m_{N}\right)\right\}, \quad(\pi N \text { loop }) \\
& R(p, k)=\frac{4}{3}\left(\frac{g_{\Delta}}{F_{\pi}}\right)^{2} F^{2}(p, k)\left\{-k^{2}+\frac{\left(p \cdot k-k^{2}\right)^{2}}{m_{\Delta}^{2}}\right\}\left(\not p-\not k+m_{\Delta}\right), \quad(\pi \Delta \text { loop })
\end{aligned}
$$

In this calculation, we consider only $N$ and $\Delta$ in the intermediate state, as we expect the contributions of higher mass resonances to be small. The reason is that on both the cuts all the distribution functions decrease rapidly with the rise of resonance mass, the only exception being $n(\omega)$ for pions on the unitary cut, which does the reverse. However, unlike the Landau cut, where all higher mass resonances can contribute, the unitary cut, for a fixed upper value of the (virtual) nucleon mass, gets contribution from only a finite number of resonances. Thus in the present case, where we restrict $p_{0} \lesssim 1.5 \mathrm{GeV}$, only $N$ and $\Delta$ can contribute to the unitary cut. 


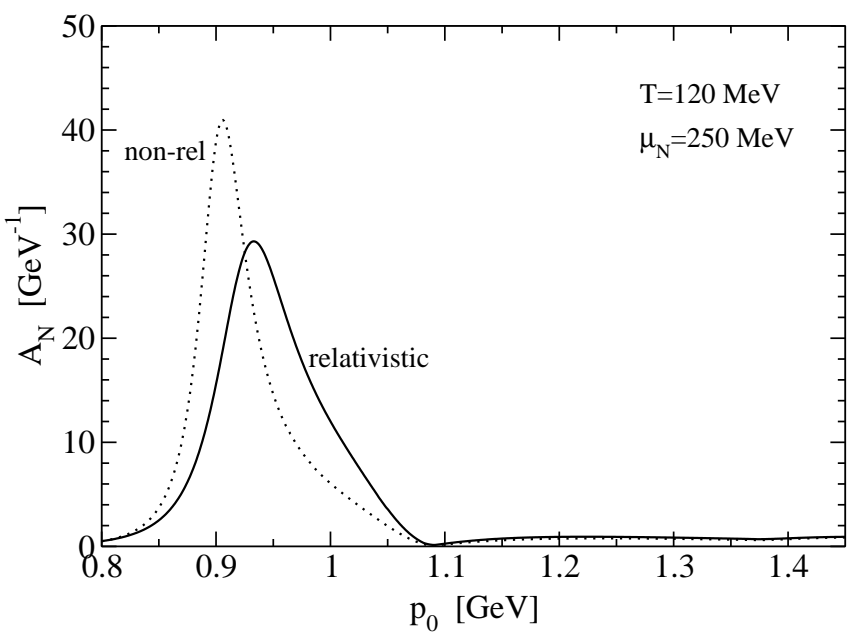

FIG. 7: Comparison of relativistic spectral function (continuous curve) with its non-relativistic limit (dotted curve).

A question arises in such calculations, whether a non-relativistic approximation could reproduce the relativistic results in a quantitative way [25]. To define this approximation, we rewrite $E^{11}\left(p_{0}, \boldsymbol{p}\right)$, the spin-independent factor in the 11-component of baryon propagator, given by Eq.(6), as

$$
E^{11}\left(p_{0}, \boldsymbol{p}\right)=\frac{-1}{2 \omega^{\prime}}\left\{\frac{1-n_{+}\left(\omega^{\prime}\right)}{p_{0}-\omega^{\prime}+i \epsilon}+\frac{n_{+}\left(\omega^{\prime}\right)}{p_{0}-\omega^{\prime}-i \epsilon}-\left(\frac{1-n_{-}\left(\omega^{\prime}\right)}{p_{0}+\omega^{\prime}-i \epsilon}-\frac{n_{-}\left(\omega^{\prime}\right)}{p_{0}+\omega^{\prime}+i \epsilon}\right)\right\}
$$

Here the first two terms describe the propagation of baryon and the last two that of antibaryon. The non-relativistic approximation to this propagator consists in retaining only the first two terms above 26, 27]. This approximation in turn gives only the first two terms in Eq. $(29)$ for $\operatorname{Re} \bar{\Sigma}\left(p_{0}, \boldsymbol{p}\right)$. Further we set $\omega^{\prime}=m$ everywhere for baryon [28]. Note that we approximate neither the propagator $D^{11}(k)$ for pion nor its energy-momentum relation.

In presenting the results of numerical evaluation, we consider, along with our relativistic framework, also the nonrelativistic one as defined above and another where the unitary cuts are switched off (by omitting the second and fourth terms in Eq. (29)), but relativistic otherwise. We note here that the values of $g_{\Delta}$ and $\Lambda$ as well as the fit to the experimental data for $\delta_{33}$ that we found earlier remain unaffected by the non-relativistic approximation. Fig. 4 compares the typical behaviour of relativistic results with the non-relativistic ones for the imaginary and real parts of self-energy, separately for the two loops - we see that only the real part for $\pi \Delta$ loop differ significantly between the two. Fig. 5 does this comparison for the real parts between the complete result and the one without the unitary cuts, showing significant difference only at higher masses. (The imaginary parts here are, of course, the same as in Fig. 4 without the steep lines representing the unitary cuts.)

Having made these comparisons, we come back to our model in Fig. 6 to draw the nucleon spectral function at different values of $T$ and $\mu$, which are realised in heavy-ion collisions [29, 30]. As expected, the height of the peak decreases with rise of temperature, while it remains about the same within the interval of chemical potential considered here [31]. Finally we again compare in Fig. 7 a typical spectral function of our calculation with its non-relativistic limit.

We also compare our results with two earlier calculations. Leutwyler and Smilga [2] use virial expansion to leading order to obtain the self-energy for on-shell nucleon in pionic medium. In Fig. 8 we compare their results for the imaginary and real parts with those from our model, setting nucleon and $\Delta$ distribution functions to zero. The good agreement shows that our model is realistic, if we recall that they evaluate the virial formula with experimental data on $\pi N$ scattering. Hees and Rapp [3] calculate the self-energy in the imaginary time formulation and takes into account only the Landau cut for the imaginary part and correspondingly only the first term in Eq. (29) for the real part. However, they take higher mass resonances in the loop, validating their model at higher temperatures and chemical potentials.

To conclude, we calculate the self-energy of the nucleon and its spectral function in the real time version of the thermal field theory in the relativistic framework. The imaginary part of the self-energy is built out of contributions from both Landau and unitary cuts from one loop graphs with $\pi N$ and $\pi \Delta$ intermediate states. In contrast to results in the literature, we find the unitary cut from the $\pi \Delta$ loop to contribute significantly in the upper region of (virtual) mass of nucleon considered. The nucleon spectral function turns out to be sensitive to non-relativistic approximation, establishing the necessity of relativistic treatment for its quantitative determination. 

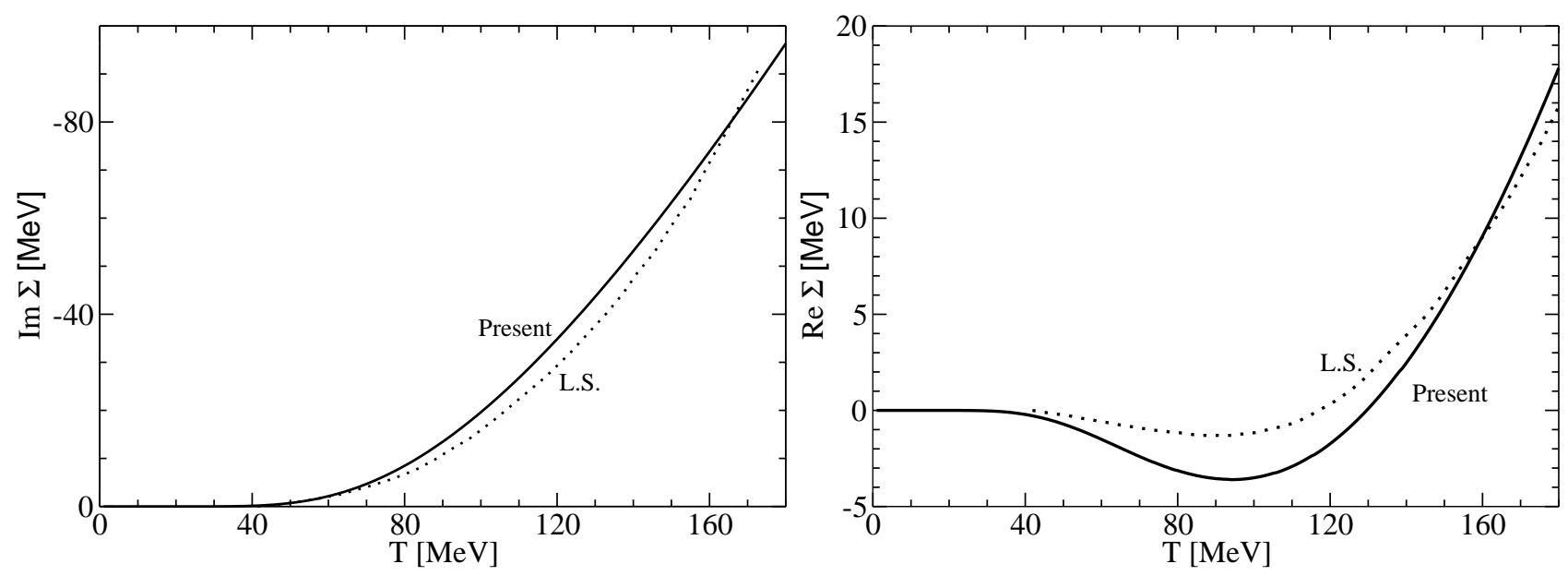

FIG. 8: The present evaluation of on-shell imaginary (left panel) and the real (right panel) parts of self-energy in pion medium (continuous curve) compared with those of Ref. [2] (dotted curve.)

\section{Acknowledgement}

One of us (S.M.) acknowledges support from Department of Science and Technology, Government of India. We also thank the Referee for his recommendations leading to improvement of the manuscript.

[1] R. Rapp and J. Wambach, Adv. Nucl. Phys. 25, 1 (2000)

[2] H. Leutwyler and A. Smilga, Nucl. Phys. B342, 302 (1990)

[3] H. van Hees and R. Rapp, Phys. Lett. B 606, 59 (2005)

[4] B. tar Haar and R. Malfliet, Phys. Rep. 149, 207 (1987)

[5] A.J. Niemi and G.W. Semenoff, Ann. Phys. 152, 105 (1984)

[6] B. L. Ioffe, Nucl. Phys. 188, 317 (1981)

[7] Y. Chung et al, Nucl. Phys. B 197, 55 (1982)

[8] The parameter $\lambda$ drops out from the self-energy function.

[9] R.L. Kobes and G.W. Semenoff, Nucl. Phys. 260, 714 (1985)

[10] S. Mallik amd S. Sarkar, Eur. Phys. J. C 61, 489 (2009)

[11] W. Rarita and J. Schwinger, Phys. Rev. 60, 61 (1941)

[12] J. Gasser and H. Leutwyler, Ann. Phys, 158, 142 (1984)

[13] G. Ecker, J. Gasser, H. Leutwyler, A. Pich and E. de Rafael, Phys. Lett. B 223, 425 (1989)

[14] T. Becher and H. Leutwyler, JHEP 0106, 017 (2001)

[15] A second term is required in $\mathcal{L}_{\pi N \Delta}$ to ensure a pure coupling to spin $\frac{3}{2}$ field [16]. In the imaginary part of the self-energy, where $\Delta$ is on mass shell, it does not contribute. But in the real part, it does, which, however, we ignore in our calculation.

[16] R.D. Peccei, Phys. Rev. 176, 1812 (1968)

[17] C.L. Korpa, R. Malfliet, Phys. Rev. C 52, 2756 (1995).

[18] W. Weinhold, B. Friman and W. Noerenberg, Phys. Lett. B 433, 236 (1998).

[19] Particle Data Group, Phys. Lett. B 667, 1 (2008)

[20] R. Koch and E. Pietarinen, Nucl. Phys. A 336, 331 (1980).

[21] S. Weinberg, The Quantum Theory of Fields, vol I, Cambridge University Press, 1995

[22] H.A. Weldon, Phys. Rev. D 28, 2007 (1983)

[23] A. Das, Finite Temperature Field Theory, World Scientific, Singapore (1998)

[24] S. Ghosh, S. Sarkar and S. Mallik, arXiv:0911 3504

[25] See for example, M. Post, S. Leupold and U. Mosel, Nucl. Phys. A 741, 81 (2004) and references cited therein.

[26] A.L. Fetter and J.D. Walecka, Quantum Theory of Many-Particle Systems, 2003, Dover publications, New York.

[27] B. D. Serot and J. D. Walecka, Adv. Nucl. Phys. 16 (1986) 1.

[28] One also approximates $\omega^{\prime}=m+(\boldsymbol{p}-\boldsymbol{k})^{2} / 2 m$, but it may lead to problems at higher momenta [26].

[29] A. Andronic, P. Braun-Munzinger and J. Stachel, Nucl. Phys. A 772 (2006) 167

[30] J. Cleymans and K. Redlich, Phys. Rev. C 60 (1999) 054908

[31] R. Rapp and C. Gale, Phys. Rev. C 60, 024903 (1999). 